\title{
Comparative Studies on Water Self-Diffusivity Confined in Graphene Nanogap: Molecular Dynamics Simulation
}

\author{
Mohammad Moulod \\ Faculty: Gisuk Hwang, PhD \\ Department of Mechanical Engineering, College of Engineering
}

Water behaviour in presence of graphene is studied in various studies and surface interaction between water and graphene is of high interest. However, confined water behaviour due to different potentials still is not completely clear. In this study, SPC/E and TIP3P water models have been confined between two fixed layers of graphene nanogaps with size of $\mathrm{Lz}=0.8$ to $4 \mathrm{~nm}$ at STP conditions. Using Molecular dynamic simulation, self-water diffusivity is calculated by the mean squared displacement approach for both lateral and vertical direction water diffusivities. It is found that the water self-diffusivity in the confined region is lower than that of the bulk water, and it decreases as the gap size decreases and the surface energy increases. No significant effect of the equilibrium distance between the water and graphene on the water selfdiffusivity is found. The in-plane water self-diffusivity is very larger than that of the out-ofplane. 